\title{
Brain network for Chinese character processing and brain computer interface
}

\author{
Xiujun $\mathrm{Li}^{1, \mathrm{a}^{*}}$, Jingjing Yang ${ }^{1, \mathrm{~b}}$ Qiyong Guo $\mathrm{o}^{2, \mathrm{c}}$ and Jinglong $\mathrm{Wu}^{3, \mathrm{~d}}$ \\ ${ }^{1}$ The School of Computer Science and Technology, Changchun University of Science and \\ Technology, 7089 Weixing Road, Changchun, Jilin, China \\ ${ }^{2}$ Department of Radiology, Shengjing Hospital of China Medical University, Sanhao Road, Heping \\ Street, Shenyang, China \\ ${ }^{3}$ The Graduate School of Natural Science and Technology, Okayama University, Tsushima-Naka \\ 3-1-1, Kita-Ku, Okayama 700-8530, Japan \\ alixiujun7897@qq.com, \\ bseiseiyou@gmail.com, ’guoqy@sj-hospital.org, 'wu@mech.okayama-u.ac.jp
}

Keywords: brain network; Chinese; brain computer interface; fMRI

\begin{abstract}
In this study, we used a 3T fMRI to investigate brain activations in processing characters and figures using visual discrimination tasks in which 13 Chinese subjects were shown two Chinese characters (36 pairs) or two figures (36 pairs). The control task (two figures) was used to eliminate the non-linguistic visual and motor confounds. The results show that discrimination of Chinese character is bilateral network for the processing of orthographic, phonological, and semantic attributes. Significant activation patterns were observed in the occipital regions (BA17,18,19,37),the temporal regions (BA22,38), the parietal regions (BA7,39,40), the frontal regions (BA4,6,10,46) and cerebellum. We conclude that constellation of neural substrates is bilateral network for Chinese subjects and used in brain computer interface (BCI).
\end{abstract}

\section{Introduction}

Recent event-related fMRI studies indicated a left-lateralized network for reading Chinese logographs processing [5]. The language can activate certain parts of our brain by either written input or spoken input. Previous neuroimaging studies by using functional magnetic resonance imaging (fMRI) or positron emission tomography (PET) have shown the different activation patterns of alphabetic language processing (e.g. English) and logographic language processing (e.g. Chinese characters) $[5,6,7,8,9,10]$. Alphabetic language information utilization preferentially involves the left inferior frontal cortex (IFC), the left medial temporal lobe (MTL), and the left temporal occipital area $[1,3]$. Chinese logographic characters differ in that they have a square configuration of similar size, packed by a number of stokes, and map onto morphemes rather than direct phonemes [10]. Accordingly an elaborative visuospatial processing is mandated in Chinese logographic system [11,12].

Language, as an important part of cognitive neuroscience, can be influenced by different socio-cultural backgrounds, while previous studies have explained that a left-lateralized network for reading Chinese words (two character Chinese words and two figures) [5]. Therefore, the pattern of interactions between the regions of the large-scale functional-anatomical network for language processing will differ during certain language tasks. As is found that different regions of the brain were activated in behavioral and functional neuroimaging studies between alphabetic subjects, the functional architecture of the brain is adjusted by literacy and education. Kuo et al report a left-lateralized network for reading Chinese words and figures using a reading task [5]. While Tan et al. reported an extensive activation of bilateral hemispheric structures in Chinese character processing, using a semantic judgment task and a homophone judgment task [10].

The present study utilizes whole-brain 3T fMRI to observe brain response during the judgment of Chinese characters and figures. The goals of the current study are threefold. First, the study inspects the commonality and particularity of brain organization for judgment Chinese relative to alphabetic languages. Second, the study exploits a control task to eliminate the non-linguistic visual and motor 
confounds. Third, the study proposes that Chinese word recognition might mandate perceptual and attention mechanisms that target the bilateral hemisphere, advantageous for local details, for the fine-grained analysis of spatial properties of Chinese characters.

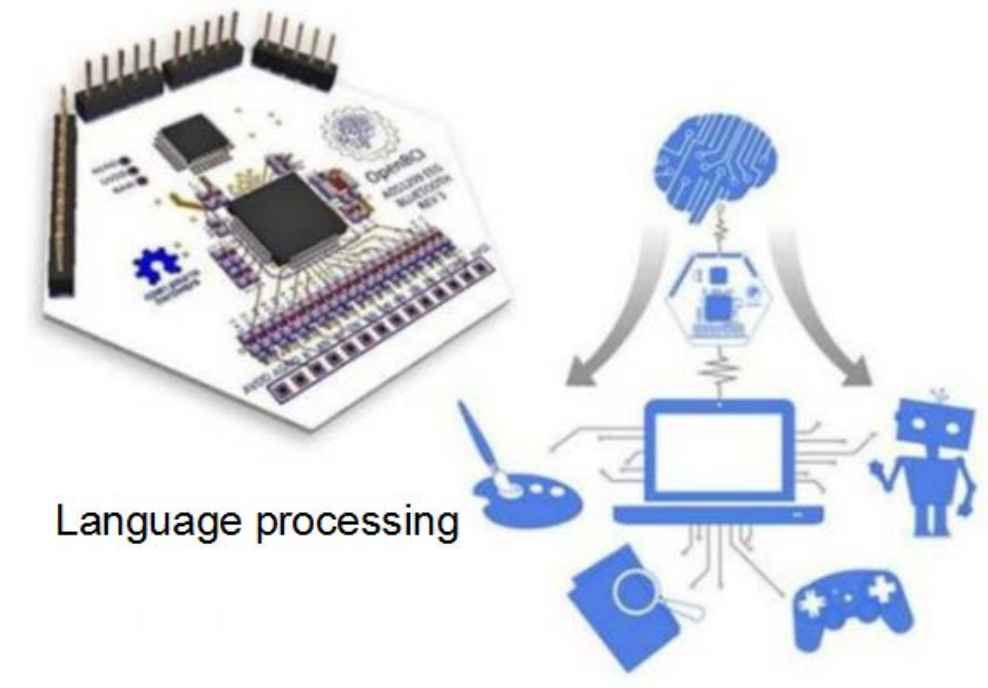

Fig.1 BCI system

\section{Methods}

\section{Subjects}

In this fMRI study, subjects were healthy Chinese by birth who grew up in the North China. All subjects were almost college teachers in Shenyang city. Thirteen right-handed participants were included in this study .All subjects averagely aged 44.38 years, 7 males, 6 females. They were selected and participated in the fMRI study with informed written consents. We explained the details of the information form and the consent form for them before we obtained their fingerprints or signing. The protocol was approved by the Ethics Committee of Shengjing Hospital of China Medical University.

\section{Task and Design}

All MRI studies were performed on a 3T Philip signal scanner at Shengjing Hospital of China Medical University (Fig. 1). 289 fMRI volumes of images were collected during each run. Each fMRI run consisted of one task. An event-retated design was used. The subjects were asked to fix on a small crosshair during the resting period. Each pair of Chinese characters or figures was shown through the projector lasting for $4000 \mathrm{~ms}$, with an interpair interval of $2000 \mathrm{~ms}, 4000 \mathrm{~ms}$ or $6000 \mathrm{~ms}$ between the stimuli. Each task consisted of half Chinese characters and half figures. The subjects were asked to the right key with their right thumbs when they judged the two characters or figures to be same, and press the left key with their left thumbs when they judged them to be different.

\section{Data Analysis}

Statistical Parametric Mapping 2 (SPM2) software and Matlab7.0 were used for the image and statistical analyses. At first, each of the 289 fMRI image volumes was automatically realigned to the first image of the time series to correct for head movements during the fMRI acquisition. Then, T1-weighted anatomical images were coregistered to the first scan in the functional images. Next, the coregistered T1-weighted anatomical images were normalized to standard T1 template images, as defined by the Montreal Neurological Institute. Finally, these spatially normalized functional images were smoothed with an isotopic Gaussian kernel of $8 \mathrm{~mm}$. Than using a one-sample t-test, activations that fell within clusters of 0 or more contiguous voxels exceeding the false discovery rate (FDR)-corrected statistical threshold of $\mathrm{P}<0.05$ were considered significant. 


\section{Results}

To In behavioral experiment: Average reaction times and response accuracy across subjects in behavioral experiments were shown in Fig. 3. Regarding the average reaction times, the control task was shorter than the language task. Regarding the average response accuracy, the control task was higher than the language task respectively.

In fMRI experiment: Significant activations have been detected during the language and control stimuli. Figure 4 showed the maps of average activation $(n=13)$ for the Chinese character judgment task and the figure judgment task respectively. Regions of significant activation during two tasks relative to the respective resting state are shown in Table 1.

The results are briefly summarized as below: during the language and control stimuli showed significant activations in: the occipital regions (BA17,18,19,37), the temporal regions (BA22,38), the parietal regions (BA7,39,40), the frontal regions (BA4,6,10,46) and cerebellum (Fig. 4 and Table 1). $(P<0.05$ Corrected cluster size $>0$ voxels $)$.

Table 1. Stereotactic Coordinates, Z Values, and Corresponding Brodmann Areas (BAs) for Regions Activated Significantly (Brain activations in the literate subjects).

\begin{tabular}{|l|c|c|c|c|c|c|}
\hline Brain areas & \multicolumn{7}{|c|}{ Coordinates } \\
\hline & BA & $\mathrm{X}$ & $\mathrm{y}$ & $\mathrm{Z}$ & $\mathrm{Ke}$ & $\mathrm{Z}$ \\
\hline Right middle occipital gyrus & $17 / 18 / 19$ & 5 & -90 & -16 & 6237 & 5.82 \\
\hline Cerebellum & & 43 & -60 & -30 & & 5.2 \\
\hline Left fusiform gyrus & 37 & -43 & -65 & -26 & & 5.09 \\
\hline Right middle occipital gyrus & $18 / 19$ & 29 & -90 & -21 & & 4.98 \\
\hline Left middle occipital gyrus & $17 / 19$ & -47 & -71 & -16 & & 4.73 \\
\hline Right middle occipital gyrus & 19 & 41 & -73 & -22 & & 4.6 \\
\hline Cerebellum & & -38 & -56 & -33 & & 4.54 \\
\hline Left middle occipital gyrus & $17 / 18$ & -3 & -89 & -21 & & 4.5 \\
\hline Left middle occipital gyrus & 18 & -22 & -93 & -21 & & 4.41 \\
\hline Right fusiform gyrus & 37 & 48 & -62 & -19 & & 4.36 \\
\hline Superior longitudinal gyrus & 4 & -40 & -7 & 49 & 87 & 4.31 \\
\hline Left middle frontal gyrus & 6 & -29 & -7 & 57 & & 3.51 \\
\hline Right superior temporal gyrus & 22 & 57 & -8 & 5 & 248 & 4.08 \\
\hline Right superior temporal gyrus & 38 & 48 & 14 & -10 & & 3.75 \\
\hline Left superior parietal gyrus & 7 & -6 & -73 & 49 & 124 & 4.07 \\
\hline Cerebellum & & 3 & -50 & -6 & 123 & 4.02 \\
\hline Right middle frontal gyrus & 10 & 41 & 39 & 25 & 155 & 3.78 \\
\hline Right middle frontal gyrus & 46 & 40 & 45 & 17 & & 3.6 \\
\hline Right superior parietal gyrus & 39 & 45 & -29 & 48 & 25 & 5.07 \\
\hline Superior longitudinal gyrus & $4 / 6$ & -13 & -21 & 67 & 58 & 4.91 \\
\hline Superior longitudinal gyrus & 4 & 3 & -27 & 63 & & 4.35 \\
\hline Left superior temporal gyrus & 38 & -48 & 6 & -7 & 75 & 4.83 \\
\hline Left middle frontal gyrus & 10 & -31 & 55 & 17 & & 4.74 \\
\hline Left middle frontal gyrus & $9 / 46$ & -20 & 61 & 19 & & 4.08 \\
\hline
\end{tabular}

\section{Discussion}

The activation of superior temporal gyri (BA22, 38) indicated the involvement of auditory cortex, as one would expect for the present task with auditory stimulation. BA47 is part of the frontal cortex in the human brain. Recent FMRI data indicate that the PFC (BA47) activity may be sensitive to the material being encoded or retrieved from long-term memory [4, 9,10,11]. It is also possible that the PFC activity is modulated by the encoding strategy used by the subjects. This may be the enhanced effects on human language processing abilities caused by literacy, which have been proved from our previous visual fMRI study on character processing. BA17, 18, 19, 37 activity may be sensitive to the visual stimulus.

Moreover, the middle frontal gyrus (BA4, 6, 10, 46) is uniquely activated in subjects. The activation of left middle frontal gyrus (BA9) has been owed to its strong relation with word encoding, 
especially with Chinese character processing $[2,9,11]$. The activation of bilateral precentral gyrus (BA6, the premortor and supplementary motor area) may be the result of quicker finger tapping, which accords to the results of behavioural experiments. About BA7, 39, 40: The activation in left superior temporal gyrus (BA39/40) and right inferior parietal lobule (BA40), as parts of Wernicke's area and its homology, are strongly associated in orthographic to phonological processing and working memory. It is probably due to the unique square spatial configuration of Chinese characters, the routine activation of which was also shown in some other verbal working memory tasks [6]. Based on such analyses, our hypothesis is confirmed that the activation regions is more related to character processing, and such a strong relation may be an acquired result by education.

\section{Summary}

In conclusion, a bilateral cortical network for Chinese character processing and this modulated brain plasticity may be acquired by education.Thanks to Jilin Provincial Education Department "Twelfth Five" Scientific and Technological Research Project (KYC-JC-XM-2015-094) for support.

\section{References}

[1] Booth, J.R., Burman, D.D., Meyer, J.R., Gitelman, D.R., Parrish, T.B., Mesulam, M.M., (2002). Functional anatomy of intra- and cross-modal lexical tasks. Neuroimage, 16: 7-22.

[2] Gabrieli, J.D., Poldrack, R.A., Desmond, J.E., (1998). The role of left prefrontal cortex in language and memory. Proc Natl Acad Sci US A, 95:906 - 913.

[3] Jobard, G., Crivello, F., Tzourio-Mazoyer, N., (2003). Evaluation of the dual route theory of reading: a metanalysis of 35 neuroimaging studies. Neuroimage, 20: 693-712.

[4] Kelley, W.M., Miezin, F.M., McDermott, K.B., Buckner, R.L., Raichle, M.E., Chohen, N.J., Ollinger, J.M., Akbudak, E., Conturo, T.E., Snyder, A.Z., Petersen, S.E., (1998). Hemispheric specialization in human dorsal frontal cortex and medial temporal lobe for verbal and nonverbal memory encoding. Neuron, 20: 927-936.

[5] Kuo, W.J., Yeh, T.C., Duann, J.R., Wu, Y.T., Ho, L.T., Huang, D., Tzeng, J.L.O., Hsieh, J.C., (2001). A left-lateralized network for reading Chinese words: a 3T fMRI study. Neuroreport, 12:3997-4001.

[6] Kuo, W.J., Yeh, T.C., Lee, J.R., Chen, L.F., Lee, P.L., Chen, S.S., Ho, L.T., Hung, D.L., Tzeng, OJ-L., Hsieh, J.C., (2004). Orthographic and phonological processing of Chinese characters: an fMRI study. NeuroImage, 21: 1721-1731.

[7] Petersson, K.M., Reis, A., Askelof, S., Castro-Caldas, A., Ingvar, M., (2000). Language processing modulated by literacy: a network analysis of verbal repetition in literate and illiterate subjects. J Cogn Neurosci, 12:364 - 382.

[8] Petersson, K.M., Reis, A., Ingvar, M., (2001). Cognitive processing in literate and illiterate subjects: A review of some recent behavioral and functional neuroimaging data. Scand J Psychol, 42:251 - 267.

[9] Tan, L.H., Liu, H.l., Perfetti, C.A., Spinks, J.A., Fox, P.T., Gao, J.H., (2001). The neural system underlying Chinese logograph reading. Neuroimage, 12: 836-846.

[10] Tan, L.H., Spinks, J.A., Feng, C.M., Siok, W.T., Perfetti, C.A., Xiong, J., Fox, P.T., Gao, J.H., (2003). Neural systems of second language reading are shaped by native language. Human Brain Mapping, 18: 158-166.

[11] Tan, L.H., Spinks, J.A., Gao, J.H., Liu, H.L., Perfetti, C.A., Xiong, J.h., Stofer, K.A., Pu, Y.l., Liu, Y.j., Fox, P.T., (2000). Brain activation in the processing of Chinese characters and words: a functional MRI study. Human Brain Mapping, 10:16-27.

[12] Tan, L.H., Chan, A.H.D., Kay, P., Khong, P.L., Yip, L.K.C., Luke, K.K., (2008). Language affects patterns of brain activation associated with perceptual decision. Proc Natl Acad Sci U S A, 105: 4004-4009. 